\title{
Grundbildung im Spannungsfeld bildungspolitischer Ein- und Abgrenzungsinteressen
}

\author{
Caroline Euringer (D)
}

Eingegangen: 29. April 2016 / Angenommen: 8. Juli 2016 / Online publiziert: 11. August 2016

(C) Der/die Autor(en) 2016. Dieser Artikel ist eine Open-Access-Publikation.

Zusammenfassung Dieser Beitrag befasst sich mit der Frage, wie der Begriff „Grundbildung“ vor dem Hintergrund von Interessen und Machtverhältnissen in der öffentlichen Bildungsverwaltung der Länder definiert wird. Mit Blick auf die New Literacy Studies werden empirisch-qualitative Ergebnisse zum Verständnis von Grundbildung und darauf bezogene Begründungslinien dargestellt. Diese wurden mittels leitfadengestützter Interviews erfasst und unter Rückbezug auf Neo-Institutionalismus und Bourdieus Ansatz zum Staat als Feld der Macht interpretiert. Die Ergebnisse zeigen, dass die Ein- und Abgrenzung des Begriffs nicht nur mit allgemeinen Zielen von Teilhabe und Weiterlernen begründet wird, sondern vor allem formale Zuständigkeiten und finanzielle Ressourcen eine Rolle dabei spielen, was als Grundbildung proklamiert wird. So dominiert vor allem ein Verständnis, das den Fokus - entgegen einem breit gedachten Grundbildungsbegriff - auf Lesen, Schreiben und Rechnen begrenzt.

Schlüsselwörter Grundbildung · Bildungsförderung · Lebenslanges Lernen · Alphabetisierung · Weiterbildungspolitik

\section{Adult basic education between interests of limitation and delimitation in education policy}

Abstract This contribution deals with the definition of adult basic education and its entanglement with interests and power relations in the field of public education administration of the German Laender. As the New Literacy Studies view literacy as a social practice that is related to interests and power relations, guided interviews concerning the definition of adult basic education as well as legitimising rationales

C. Euringer $(\bowtie)$

Universität Hamburg, Hamburg, Deutschland

E-Mail: caroline.euringer@uni-hamburg.de 
from the perspective of education administration were illustrated. The interviews are based on a subject-logical approach and interpretations are linked to Neo-Institutionalism and Bourdieu's theory on the state as a field of power. The findings show that the definition of adult basic education is not only grounded in general objectives such as participation and learning, but also responsibilities and financial rationales play a significant role in shaping the concept of adult basic education. Thus, dominant concepts of adult basic education are - in contrast to broader definitions limited to reading, writing and numeracy.

Keywords Adult basic education $\cdot$ Subsidies $\cdot$ Lifelong learning $\cdot$ Literacy $\cdot$ Adult education policy

\section{Einleitung}

Im Zuge der derzeitigen Diskussion zum Grundbildungsbegriff wird vielfach das Fehlen eines einheitlichen, Akteure und Felder übergreifend anerkannten Verständnisses von „Grundbildung“ betont. Sichtbar wird ein Interesse der am Diskurs beteiligten Akteure an einem Austausch darüber, was eine Grundbildung Erwachsener umfasst und wie sie sich von anderen Begriffen, z. B. „Allgemeinbildung“ oder ,Erwachsenen- und Weiterbildung“, unterscheidet (vgl. Egloff 2014, S. 104). So finden sich zunehmend Bestrebungen, über Zusammenschlüsse, Gremien und Strategien einen gemeinsamen Austausch anzuregen. Auf bildungspolitischer Ebene belegen die von Bund und Ländern gemeinsam initiierte nationale Strategie für Alphabetisierung und Grundbildung Erwachsener (BMBF und KMK 2012) sowie deren Überführung in eine nationale Dekade die bildungspolitische Relevanz dieses Themas. Flankiert wird dies durch eine Reihe von Fachtagungen und Arbeitstreffen zur begrifflichen Schärfung des Gegenstandes. ${ }^{1}$

Im Kontext der aktuellen Situation von Migration, Flucht und Asyl wird in der Diskussion zu der Frage, was denn Grundbildung sei, gefordert, eine begründete Einund Abgrenzung des Grundbildungsbegriffs vorzunehmen: Lässt sich die kulturelle und politische Weiterbildung für Zuwanderer und Geflüchtete ebenfalls unter dem Grundbildungsbegriff subsumieren? Ist die Vermittlung von Sprach- bzw. Schriftsprachkenntnissen, wie sie in den vom Bundesamt für Migration und Flüchtlinge (BAMF) geförderten Integrationskursen geschieht, als Grundbildung zu betrachten und folglich auch als Aufgabe der in einigen Bundesländern gegründeten Grundbildungszentren? In welchem Verhältnis stehen die Ministerien und Ressorts, die auf Bund- und Länderebene für die Förderung von Angeboten bezüglich Grundbildung versus Migration und Flucht, aber auch beispielsweise Arbeit und Beruf zuständig sind?

\footnotetext{
1 So beispielsweise die Fachtagung „Grundbildung: Definition - Themenfelder - Zielgruppen? Der Versuch einer Begriffsbestimmung“, die am 20.04.2016 vom Rat der Weiterbildung-KAW und dem Deutschen Institut für Erwachsenenbildung (DIE) in Berlin durchgeführt wurde und einen Austausch zwischen Praxis, Wissenschaft und Politik anstrebte (vgl. Rat der Weiterbildung-KAW 2016).
} 
Zumindest im internationalen Diskurs wird bereits seit Längerem gefordert, Grundbildung als ein Querschnittsthema über bestehende Zuständigkeitsgrenzen hinweg zu betrachten (vgl. Mallows und Carpentieri 2015). Gleichzeitig werden allerdings auch Befürchtungen geäußert, die sich auf die infrastrukturellen und finanziellen Konsequenzen einer Ausweitung von Grundbildung beziehen (vgl. Linde 2002, S. 22). Zudem zeichnet sich in Diskussionen auf Fachtagungen die Sorge ab, es könne angesichts knapper öffentlicher Mittel zu einer Ablösung bzw. Begrenzung der Erwachsenenbildung durch den auf ein Minimum reduzierten Begriff der Grundbildung kommen. Die hinter der Ein- und Abgrenzung des Grundbildungsbegriffs liegenden Interessen der beteiligten Akteure, insbesondere der für die Förderung zuständigen Bildungspolitik und -verwaltung, sind bislang empirisch noch nicht untersucht worden.

Gegenstand dieses Beitrags ist es, die Verwobenheit des Begriffs Grundbildung mit Interessen und Machtverhältnissen am Beispiel der öffentlichen Bildungsverwaltung herauszuarbeiten und in ihren Konsequenzen für die Ein- und Abgrenzung des Begriffs zu beleuchten. Gerade den in den Landesregierungen angesiedelten (Weiter-)Bildungsressorts obliegt angesichts der Kulturhoheit der Länder die Umsetzung der förderpolitischen Rahmenbedingungen für Grundbildung.

Im Folgenden wird dazu zunächst ein Einblick in aktuelle Begriffsdefinitionen gegeben. Im Anschluss an die New Literacy Studies wird Grundbildung als ein interessengebundener und in Machtstrukturen eingebetteter Begriff verstanden. Bezugnehmend auf Neo-Institutionalismus und Bourdieus Ansatz zum Staat wird die öffentliche Bildungsverwaltung theoretisch gerahmt als ein Feld, in dem Interessen und Machtverhältnisse durch das Handeln und Entscheiden der Akteure reproduziert werden. Auf der Basis von leitfadengestützten Interviews zum Grundbildungsverständnis der öffentlichen Bildungsverwaltung wird die dort vorgenommene Ein- und Abgrenzung des Begriffs mit Blick auf Interessen und Machtverhältnisse schließlich begründungslogisch betrachtet und diskutiert.

\section{Grundbildung im Spannungsfeld von Interessen und Macht}

Der Diskurs zu Grundbildung in der deutschen Erwachsenen- und Weiterbildung zeichnet sich durch die Betonung einer Offenheit, Relativität und Dynamik des Begriffs bei gleichzeitigem Vorliegen einer - fast schon an Beliebigkeit grenzenden, weil proklamierten - Vielfalt unterschiedlicher, als legitim angesehener Konzepte aus (vgl. Euringer 2016a, S. 18 ff.). So wird die Frage, was eine Grundbildung Erwachsener umfasse bzw. nicht umfasse, zunächst angesichts unterschiedlicher gesellschaftlicher und individueller Anforderungen als kontextbezogen, veränderlich und nie abgeschlossen betrachtet. Grundbildung meint demnach

die Minimalvoraussetzungen an Wissensbeständen, Kenntnissen, Fertigkeiten, personalen und sozialen Kompetenzen, die für Orientierung und aktives Handeln in der Gesellschaft notwendig sind (...). Grundbildung ist vom inhaltlichen Umfang her kein feststehend definierter Begriff, da gesellschaftliche Anforderungen und individuelle Lebenslagen steter Entwicklung unterliegen (Döbert 1999). 
Welche Inhalte und Kompetenzen als mindestens hinreichend gelten, erklärt sich danach, was für Orientierung und aktives Handeln vorausgesetzt wird. Nach Tenorth stellt Grundbildung zudem die unterste Stufe bzw. das Minimum sich anschließender Bildungs- und Lernprozesse dar (vgl. Tenorth 2004, S. 170).

Derzeit existieren diverse Modelle und Konzepte, die sich im Hinblick auf die Semantik des Begriffs Grundbildung deutlich voneinander unterscheiden, aber jeweils für sich Angemessenheit und Gültigkeit beanspruchen. Mindestens drei Kontroversen zur Ein- und Abgrenzung des Begriffs lassen sich hier unterscheiden (vgl. Grotlüschen et al. 2014, S. 54 f.). Während die Kontroverse um die Kanonisierung des Begriffs nach den Inhalten und Kompetenzen einer Grundbildung Erwachsener fragt und dabei vor allem die Abgrenzung von Grundbildung zur „Alphabetisierung“, „Allgemeinbildung“ sowie zur „Erwachsenen- und Weiterbildung“ thematisiert, geht es bei der Hierarchisierungs- und Minimumkontroverse um die Bestimmung eines als hinreichend betrachteten Mindestniveaus dieser Inhalte und Kompetenzen. Zu ergänzen wäre hier eine weitere Kontroverse, die sich mit der interessenbezogenen Ziel- und Zweckbestimmung von Grundbildung befasst und somit die Legitimation der Ein- und Abgrenzung von Grundbildung thematisiert (vgl. Euringer 2016a, S. $210 \mathrm{ff}$.).

Vor dem Hintergrund dieser pluralen Sichtweisen auf Grundbildung spricht Tröster (2000) von einem „Spannungsfeld Grundbildung“. Sie versteht Grundbildung als einen relationalen Begriff, der im Spannungsfeld der Ansprüche und Interessen verschiedener Akteure und Gruppen stehe (vgl. ebd., S. 18). Die Ein- und Abgrenzung von Grundbildung sei eine Frage der Perspektive und demnach vielfältig und offen. Was hier allerdings vernachlässigt wird, ist die Existenz gesellschaftlicher Machtverhältnisse, die zur ungleichen Durchsetzung von Interessen beitragen. Anschlussfähig ist diese Sichtweise an die soziokulturelle Theorietradition der New Literacy Studies, die „Literacy“ nicht nur als kognitive Kompetenz, sondern als soziale Praxis verstehen, die in Werte, Einstellungen und Wissensbestände des soziokulturellen Kontexts eingebettet ist. Grundbildung variiert folglich von Kontext zu Kontext und auch die Sichtweisen auf Grundbildung sind immer ideologisch, weil sie an die Interessen bestimmter Akteure rückgebunden sind (vgl. Street 1992; Barton und Hamilton 2000). Vor allem mächtige soziale Akteure, wie z. B. staatliche Institutionen, können bestimmte Sichtweisen auf Grundbildung als gesellschaftlich wertvoll durchsetzen und damit zugleich andere Sichtweisen abwerten und marginalisieren (vgl. ebd., S. 11 ff.). Bereits Bourdieu (2005) weist auf die Macht einer „legitimen Sprache“ hin und Grotlüschen, Heinemann und Nienkemper konstruieren entsprechend das Pendant einer ,legitimen Literalität“, welche die Interessen der Oberschicht reproduziert (vgl. Grotlüschen et al. 2009).

Wird Grundbildung im Anschluss an Tröster also als ein Konzept verstanden, welches vielfältig, offen und dynamisch ist und sich im Spannungsfeld unterschiedlicher Interessen und Ansprüche definiert, so muss hier ergänzt werden, dass sich vor allem solche Sichtweisen auf Grundbildung im Diskurs als legitim durchsetzen, welche die Interessen mächtiger sozialer Akteure repräsentieren. Ebenfalls ist davon auszugehen, dass Sichtweisen auf Grundbildung, die von weniger mächtigen Akteuren vertreten werden, eine untergeordnete, weniger dominante Rolle einnehmen. 


\section{Theoriezugänge zum bildungspolitischen Feld}

Ausgehend von der Annahme, dass die Ein- und Abgrenzung von Grundbildung mit Interessen und Machtverhältnissen verwoben ist, wird eine theoretische Perspektive auf Bildungspolitik und -verwaltung eingenommen, welche die dort wirksamen Normen, Regeln, Institutionen und Praktiken kritisch in den Blick nimmt: Wie werden Entscheidungen getroffen? Welche Bedeutung haben dabei der gesellschaftliche und institutionelle Kontext sowie die darin eingebetteten Praktiken, Interessen und Machtverhältnisse?

Grundsätzlich bedeutsam für das Handeln in der öffentlichen Verwaltung sind Gesetze und rechtliche Normierungen, die den Rahmen für den Vollzug politischer Vorgaben bilden (vgl. Kegelmann 2013, S. 248). Bezogen auf Erwachsenenund Weiterbildung übernehmen neben den Bildungsfreistellungs- und Schulgesetzen insbesondere die in den meisten Bundesländern vorhandenen Erwachsenenbildungsbzw. Weiterbildungsgesetze eine strukturbildende Funktion, indem sie das Verhältnis des Staats zu den Einrichtungen und Trägern der Erwachsenen- und Weiterbildung sowie Fragen zur Programmatik, Organisation, Finanzierung und Qualität von Weiterbildung regeln (vgl. Faulstich und Haberzeth 2007, S. 57). Die Förderung von Grundbildung als Teil der allgemeinen Weiterbildung ist dabei in einigen Erwachsenenbildungsgesetzen explizit begrifflich enthalten oder implizit möglich, indem über einen Negativkatalog eine Förderung von Grundbildung nicht grundsätzlich ausgeschlossen ist. Folglich ist ein gewisser Raum zur Ausgestaltung von Grundbildung gegeben.

Über diese formalen Richtlinien hinaus ist die öffentliche Bildungsverwaltung zudem als ein „System von Institutionen“ (Jann 2008, S. 17) bzw. institutionellen Regeln zu verstehen, welche die Auslegung der Gesetze und rechtlichen Normierungen strukturiert. Der Neo-Institutionalismus, der die Schnittstelle von Gesellschaft und Organisation betrachtet, geht davon aus, dass gesellschaftliche Institutionen als soziale Regeln das organisationale Handeln bestimmen, indem sie einen Legitimationskontext konstituieren, der mehr oder weniger bewusste Normalitätsvorstellungen hinsichtlich der sozialen Welt enthält. Nach Koch bestimmt der gemeinsam geteilte, vorstrukturierte „legitimatorische Kontext organisationalen (Steuerungs-)handelns“ (Koch 2011, S. 85) die (il-)legitimen Handlungsmöglichkeiten und -spielräume. Auch internationale Institutionen können so Einfluss auf nationale Politiken ausüben (vgl. Schemmann 2007; Ioannidou 2010).

Dass die Aushandlung legitimer sozialer Institutionen zudem keineswegs unabhängig von Interessen und Machtverhältnissen geschieht, darauf verweist Bourdieu in seinen Ausführungen zum Staat. Bourdieu versteht Staat und öffentliche Verwaltung als Inhaber des „Monopols der legitimen physischen und symbolischen Gewalt“ (Bourdieu 2014, S. 18), da sie die offizielle legitime symbolische Ordnung in der Gesellschaft festlegen und bestimmen, was als ,richtige“ bzw. legitime Sicht auf soziale Welt gilt. Diese Benennungsmacht stellt eine wichtige Machtquelle dar, weil die Durchsetzung von Repräsentationen als eine „Herrschaft über das Denken und über die Köpfe“ (Bremer 2010, S. 183) verstanden werden kann. Um eine solche Macht allerdings ausüben zu können, müsse sich der Staat als Hüter des Allgemeinen ausgeben: als „standpunktloser Standpunkt“ (Bourdieu 2014, S. 61) über den 
unzähligen Partikularinteressen, der im Sinne einer übergreifenden Instanz seine Interessen im Namen der Allgemeinheit oder des öffentlichen Willens formuliert. Durch dieses „Spektakel des Allgemeinen“ gelinge laut Bourdieu das ,,alchimistische Kunststück (...), Besonderes in Allgemeines zu verwandeln“ (ebd., S. 71) und nur so sei gewährleistet, dass die zur Ausübung von Macht notwendige Verkennung ihres Herrschafts- und Willkürcharakters verkannt bzw. die Macht von den Beherrschten selbst anerkannt und reproduziert werde. Der Staat diene somit zur Aufrechterhaltung der öffentlichen Ordnung zugunsten der Herrschenden, die ihre Interessen im Rahmen von Machtkämpfen als legitim durchsetzen konnten (vgl. ebd., S. 68).

\section{Methodik: Leitfadengestützte Interviews}

Zur empirischen Analyse des Verständnisses von Grundbildung in der öffentlichen Bildungspolitik wurde ein subjektlogischer Zugang gewählt, der mittels qualitativer Leitfadeninterviews mit Schlüsselakteuren der öffentlichen Bildungsverwaltung eine komplexe Annäherung an das Zusammenwirken von Grundbildung, Interessen und Macht ermöglicht. Ausgangspunkt hierfür stellt die von Holzkamp (1985, 1995) subjektwissenschaftlich fundierte Handlungstheorie dar, mit welcher es gelingt, das Handeln gesellschaftlich eingebetteter Subjekte von ihrem je eigenen Subjektstandpunkt her zu erfassen und dabei zugleich die gesellschaftlichen Bedingungen und Machtverhältnisse, zu denen sich die Subjekte in ihrem Handeln verhalten, nicht außer Acht zu lassen. In Abgrenzung zu einer einseitigen Verabsolutierung bzw. Objektivierung betont Holzkamp, dass Subjekte trotz ,gesamtgesellschaftlicher Vermitteltheit ihrer Existenz“ (Holzkamp 1985, S. 342) prinzipiell die Fähigkeit besitzen, über die eigenen Lebensumstände zu verfügen. Das Subjekt verhält sich zu diesen ,als ein sinnlich-körperliches, bedürftiges, interessiertes Subjekt“ (Holzkamp 1995, S. 21, Herv.i.O.), wodurch die soziale Welt vermittelt in ihrer Bedeutung für das Subjekt in den Blick gerät. Handlungen und Entscheidungen - und damit auch die Ein- und Abgrenzungshandlungen im Hinblick auf Grundbildung - sind ,in den Lebensbedingungen ,begründet““ (Holzkamp 1985, S. 348) und nur vor diesen intersubjektiv nachvollziehbar.

Es wurden zwölf telefonische Leitfadeninterviews mit Schlüsselakteuren aus der öffentlichen Bildungsverwaltung der Länder geführt und durch sechs weitere Interviews mit Akteuren aus dem Feld der Alphabetisierung und Grundbildung kontrastiert. Die Auswahl der Befragten aus der öffentlichen Bildungsverwaltung erfolgte dabei nach den formalen Zuständigkeiten für Grundbildungspolitik. ${ }^{2}$

In einem möglichst offenen Gespräch wurden die Akteure nach dem Verständnis von Grundbildung (,Was ist Grundbildung?“) sowie nach den Begründungsmustern (,Warum? Wozu?“) gefragt. Dabei galt es angesichts zeitlicher Restriktionen, eine

\footnotetext{
2 Angesichts der nur kleinen und öffentlich exponierten Grundgesamtheit wird aus Gründen der Anonymitätswahrung an dieser Stelle nicht weiter auf die Fallauswahl eingegangen, bei der beispielsweise auch strukturelle Merkmale wie Flächen- bzw. Stadtstaat oder das Vorhandensein bzw. Fehlen eines Erwachsenenbildungsgesetzes eine Rolle spielte.
} 
angemessene Balance zwischen dem forschungsleitenden Interesse und Vorwissen der Interviewerin einerseits und dem Wissen und den Relevanzen der Interviewten andererseits zu schaffen. Zur Vermeidung der dem Leitfadeninterview inhärenten Gefahr einer „Leitfadenbürokratie“ (Hopf 1978), welche die Relevanzen der Interviewten nicht angemessen berücksichtigt, wurden offene, erzählgenerierende sowie vertiefende Fragen eingesetzt (vgl. Mey und Mruck 2010, S. 430). Eine wesentliche Herausforderung bestand darin, Raum auch für (noch) nicht reflektierte, wenig offenkundige sowie komplexe und kontroverse Sichtweisen zu schaffen, ohne die Gesprächsführung durch eigene Kategorien und Konzepte übermäßig zu steuern.

Die auf diese Weise geäußerten Sichtweisen auf Grundbildung wurden im Anschluss an Holzkamp als Ergebnis eines im gesellschaftlich-institutionellen Bedingungsgefüge begründeten Subjekthandelns verstanden. Die Auswertung erfolgte in Anlehnung an die drei Kodierschritte (offenes, axiales, selektives Kodieren) der Grounded Theory (Strauss und Corbin 1996), wobei dem axialen Kodieren ein begründungslogisch gewendetes Kodierparadigma zugrunde gelegt wurde. ${ }^{3}$ Ausgehend vom Standpunkt des interviewten Subjekts lassen sich die deterministisch konnotierten Begriffe Kontext, ursächliche Bedingungen, intervenierende Bedingungen sowie Konsequenzen nur jeweils in ihrer Bedeutung für das Subjekt begründen und müssen folglich auch aus dieser Perspektive nachvollzogen werden (vgl. Euringer 2016a, S. 128):

(A) Bedeutungskonstellation im subjektiven Möglichkeitsraum,

(B) Prämissen,

(C) Grundbildungsverständnis,

(D) intendierte Konsequenzen.

Das von den Interviewten jeweils geäußerte Verständnis und deren Begründungen wurden schließlich mithilfe des Kodierparadigmas in einen begründungslogischen Zusammenhang gebracht, wobei untersucht wurde, welche Interessen zur Begründung der Grundbildungsdimensionen herangezogen wurden und ob hier systematische Gemeinsamkeiten und Unterschiede erkennbar sind.

\section{Grundbildung als Folge von Ein- und Abgrenzungsinteressen}

Die Auswertung der Interviews zeigt sehr deutlich, dass über die einzelnen Bundesländer hinweg Grundbildung entlang von vier Dimensionen (Inhalte und Kompetenzen, Mindestniveaus, Zielgruppen, Angebotsformate) spezifiziert wird, allerdings kein einheitliches Verständnis einer Grundbildung Erwachsener besteht. Vielmehr wird die Frage, was Grundbildung umfasse, vor dem Hintergrund der jeweils gegebenen förderpolitischen Rahmenbedingungen beantwortet und mit Verweis auf unterschiedliche Ziele und Interessen begründet (vgl. Euringer 2016b, S. 37 ff.). Es zeigt sich eine Begründungslogik, die mit Bourdieu als ,Spektakel des Allgemeinen“ (Bourdieu 2014, S. 71) bezeichnet werden kann und sich darin äußert, dass allgemeine, das öffentliche Wohl betreffende Ziele betont werden, die ein gemein-

\footnotetext{
3 Eine ähnliche begründungslogische Wendung des Kodierparadigmas findet sich beispielsweise auch bei Anke Grotlüschen (2010) und Barbara Nienkemper (2015).
} 
sames Interesse vorzugeben scheinen. In den Interviews wird hier vor allem auf gesellschaftliche und berufliche Teilhabe, die Bewältigung des Alltags sowie die Befähigung zum Weiterlernen im Sinne Lebenslangen Lernens verwiesen, zu welcher eine breit gedachte Grundbildung befähigen solle. Tatsächlich zeigt sich aber, dass vor allem partikulare Ein- und Abgrenzungsinteressen für die Entscheidung, was förderpolitisch als Grundbildung gilt, handlungsleitend sind und unter dem Deckmantel eines breit gedachten Begriffs den Fokus im Wesentlichen auf Lesen, Schreiben und Rechnen begrenzen.

\subsection{Ermöglichung von Teilhabe und Weiterlernen}

In den Interviews zeigt sich eine Ausrichtung bzw. Orientierung des Grundbildungsverständnisses an allgemeinen Partizipationszielen. Was eine Grundbildung Erwachsener umfasst, wird daran bemessen, ob es ,die Voraussetzung für eine gleichberechtigte, eigenverantwortliche Teilhabe am gesellschaftlichen, kulturellen und beruflichen Leben“ (Interview 03, Z. 02) schafft. Auch politische Teilhabe im Sinne von Staatsbürgerschaft wird genannt. Zudem stellt die Befähigung zum (lebenslangen) Weiterlernen eine zentrale Legitimationslinie von Grundbildung dar. Grundbildung umfasst demnach die „Bildung, die man zuerst haben muss, um zumal fähig zu werden, fähig zu sein, sich überhaupt fortzubilden“ (Interview 04, Z. 24). Für die Frage der Ein- und Abgrenzung von Grundbildung hat dies zur Folge, dass Grundbildung „mehr“ als Lesen und Schreiben umfasst. Es wird betont, dass weitere Kompetenzen notwendig seien, die über eine klassische Alphabetisierung hinausgingen. Genannt werden Rechenfähigkeit, finanzielle Grundbildung, Umgang mit neuen Medien, politische Grundkompetenzen, gesundheitsbezogene Grundkenntnisse, Lernen lernen, Sozial- und Selbstkompetenzen sowie Englisch und Deutsch als Fremd-/Zweitsprache, wobei deutliche Unterschiede in der Ausdeutung dieser Inhalte und Kompetenzen zwischen den interviewten Akteuren erkennbar sind.

Ein ähnlich breites Konzept grundlegender Kompetenzen existiert bereits mit den „Schlüsselkompetenzen für Lebenslanges Lernen“ (Europäische Kommission 2007). Auf diese wurde von den Interviewten allerdings nur vereinzelt explizit verwiesen.

Kommen allerdings verstärkt förderpolitische Aspekte in den Blick, so zeigt sich, dass das breite Verständnis von Grundbildung sukzessive relativiert und reduziert wird. So gilt: „Förderpolitisch müsste man das natürlich unterscheiden, was zu einer Grundbildung gehört“ (Interview 06, Z. 34). Das breite, über Lesen und Schreiben hinausgehende Grundbildungsverständnis reduziert sich angesichts förderpolitischer Notwendigkeiten und Interessen und entgegen der auf Teilhabe und Weiterlernen ausgerichteten Begründungslogik insbesondere auf die alltagsbezogene Anwendung von Lese-, Schreib- und Rechenkompetenzen, die als notwendiger Kern einer Grundbildung Erwachsener priorisiert werden. Zur Begründung dieser Ein- und Abgrenzung werden drei Interessen herangezogen: Einhaltung formaler Zuständigkeiten, Sicherstellung ausreichender Finanzierung sowie Nachweis erfolgreicher Förderung über Teilnahmezahlen. 


\subsection{Einhaltung formaler Zuständigkeiten}

Aus förderpolitischer Perspektive wird Grundbildung ein- und abgegrenzt, um den mit dem Grundbildungsbegriff einhergehenden öffentlichen Förderanspruch möglichst begrenzt zu halten, denn: „Nicht alle können alles fördern. Also muss klar sein, wer welche Zuständigkeiten hat" (Interview 05, Z. 98). Dazu wird der Zuständigkeitsbereich zum einen mit Blick auf gesetzliche Vorgaben wie beispielsweise Erwachsenen- und Weiterbildungsgesetze eingegrenzt, zum anderen über Zuständigkeiten und Verantwortlichkeiten anderer Ministerien und Ressorts abgegrenzt. Die Weiterbildungsgesetze lassen zwar in der Regel offen, was konkret unter einer Grundbildung zu verstehen und zu fördern ist; allerdings wird ein gesamtgesellschaftlicher Nutzen, eine deutliche Orientierung am bestehenden Angebot der öffentlich geförderten Träger sowie eine Fokussierung auf die Zielgruppe der Bildungsfernen betont.

Über die Abgrenzung zu den Schulressorts, den Arbeitsministerien und Jobcentern sowie dem BAMF findet eine sehr klare Begrenzung bestimmter Inhalte, Kompetenzen und Formate statt. Besonders deutlich wird dies beispielsweise anhand nachholender Schulabschlüsse, die in den zusätzlich geführten Kontrastinterviews mit weiteren Schlüsselakteuren immer wieder als wichtiger Bestandteil einer grundlegenden Bildung genannt werden, von den Interviewten aus der öffentlichen Bildungsverwaltung allerdings sehr unterschiedlich aufgrund formaler Zuständigkeiten und Verantwortlichkeiten gehandhabt werden:

Die Schulabschlüsse sind von der Verantwortlichkeit hier im Kultusministerium angesiedelt, gehören aber nicht in den Bereich der Weiterbildung, sondern fallen unter das Schulgesetz. Und das ist eine ganz andere Ebene (...). Inhaltlich hat das schon Berührungspunkte, (...) aber von der Organisation und Finanzierung ist es völlig getrennt (Interview 07, Z. 50-54).

Im Unterschied dazu wird in einem anderen Interview der zweite Bildungsweg hingegen als Teil einer breiter gedachten Grundbildung Erwachsener betrachtet, da die Förderung gerade nicht, wie es in manch anderen Bundesländern der Fall sei, im staatlichen Schulsystem verortet ist (vgl. Interview 09, Z. 60). Ähnliche Abgrenzungsdiskurse finden sich bezogen auf die berufliche Nachqualifizierung, die insbesondere der Bundesagentur für Arbeit und den Jobcentern obliegt, sowie bezogen auf die vom BAMF finanzierten Sprach- und Integrationskurse für Zugewanderte. Auch hier kann eine inhaltliche Zugehörigkeit zum Grundbildungsbegriff von einer formalen Abgrenzung aufgrund fehlender Zuständigkeit unterschieden werden:

Selbstverständlich sind die Länder auch im Bereich der Integrationskurse sehr aktiv. Dazu würden wir jetzt zahlenmäßig uns gar nicht weiter verhalten wollen, weil das Zuständigkeiten im Bereich des Ressorts des Inneren sind. Und in dem Bereich wird viel gemacht, aber es würde jetzt hier unsere Zuständigkeit in der Weiterbildung glaube ich weit übersteigen, wenn wir jetzt auch noch anfangen würden, hier weiter zu gucken (Interview 05, Z. 94).

Dies führt zu der paradoxen Situation, dass Deutsch als Fremd-/Zweitsprache zwar durchaus inhaltlich als Teil einer Grundbildung Erwachsener angesehen wird, 
weil es eine wichtige Rolle im Hinblick auf Teilhabe und Weiterlernen für Zugewanderte spielt. Aufgrund der Trennung der förderpolitischen Zuständigkeiten - Länder für Weiterbildung, BAMF für Integrationskurse - kommt es allerdings zu einer Ausklammerung dieser überwiegend formalen Angebotsformate aus dem Grundbildungsverständnis der öffentlichen Bildungsverwaltung; Letztere ist im Rahmen der nationalen Strategie für die Förderung von Grundbildung zuständig.

\subsection{Sicherstellung ausreichender Finanzierung}

Eine weitere Begründungslinie zur Ein- und Abgrenzung des Grundbildungsverständnisses wird im Hinblick auf die Sicherstellung einer ausreichenden Finanzierung angesichts knapper Gelder geäußert: „Also es läuft ja im Prinzip nichts ohne Geld“ (Interview 03, Z. 116). Dies führt zu dem Interesse, den Grundbildungsbegriff auf einen minimalen Kern von Fähigkeiten - insbesondere Lesen, Schreiben und Rechnen - einzugrenzen, um dem damit verbundenen finanziellen Förderanspruch gerecht werden zu können:

Aber wer muss das bezahlen? Diese Frage ist, glaub ich, eine, die ganz oft nicht gestellt wird und nicht diskutiert wird, aber die unterschwellig mitläuft (...). Und insofern gibt es natürlich auch da Bemühungen, dass es begrenzt wird, damit es bezahlbar bleibt. Aber ohne das so auszusprechen, weil ich glaube, viele haben auch Angst, solche Diskussionen zu führen. Das ist ja nicht opportun zu sagen: Ja, es gibt Grenzen. Sondern man möchte ja eher sagen: Ja, das ist wichtig und selbstverständlich und wenn das Voraussetzung für Teilhabe ist (Interview 10, Z. 46).

Auch die Versuche, die Landesmittel zu erhöhen oder weitere Mittel einzuwerben, beispielsweise über den Europäischen Sozialfond (ESF) oder den Einbezug von Unternehmen, werden als Interessen geäußert. Offenbar spielt zur Mitteleinwerbung auf Ebene der Landespolitik der Rückbezug auf (inter-)nationale Kompetenzstudien, wie z. B. die nationale leo.-Studie (Grotlüschen und Riekmann 2012), eine wichtige Rolle, befördert aber ebenfalls eine Verengung von Grundbildung auf Lese- und Schreibkompetenzen:

Die Leute, auf die es ankommt, die zum Beispiel entscheiden, dass man Geld für etwas bekommt oder dass man Zugänge bekommt, die sind ja alarmiert durch die leo.-Studie. Und da geht es um funktionalen Analphabetismus. Das muss man ja bedenken, die Entscheider sind ja im Regelfall überhaupt nicht vom Fach. Und Grundbildung ist dann ein Begriff, der von denen überhaupt nicht wiedererkannt wird (Interview 11, Z. 16).

Mittels der Ko-Finanzierung durch den ESF, der den Abbau von Benachteiligung am Arbeitsmarkt zum Ziel hat, findet zudem eine Stärkung der arbeitsplatzbezogenen Alphabetisierung und Grundbildung statt, wie sie auch derzeit vom Bundesministerium für Bildung und Forschung (BMBF) priorisiert wird. Ziel einer arbeitsplatzbezogenen Grundbildung ist die Sicherung bzw. Stabilisierung und Verbesserung der Beschäftigungsfähigkeit sowohl von Erwerbslosen als auch Beschäftigten. 


\subsection{Nachweis erfolgreicher Förderung über Teilnahmezahlen}

Neben der Ein- und Abgrenzung des Grundbildungsbegriffs entlang von Förderzuständigkeit und Finanzierung wird zudem das Interesse geäußert, Grundbildung auf solche Inhalte, Kompetenzen und Zielgruppen zu begrenzen, die eine erfolgreiche Förderung von Angeboten ermöglichen: „Wir können Hunderte von Kursen anbieten, aber uns fehlen die Menschen, die die Kurse besuchen“ (Interview 04, Z. 74).

Hintergrund dafür ist der durch Politik aufgebaute Druck, eine erfolgreiche Förderung von Grundbildung zu gewähren und dies über Indikatoren, Kennzahlen und Benchmarks sichtbar zu machen. Für das Grundbildungsverständnis der öffentlichen Bildungsverwaltung hat dies zur Folge, dass die Förderung zunehmend auf die antizipierten Interessen leicht zugänglicher Zielgruppen ausgerichtet wird:

Es ist eng, wenn wir nur darüber sprechen, dass die Menschen ausreichend lesen und schreiben können müssen. Damit ist es nicht möglich, einen Zugang zu bekommen und Menschen zu motivieren, sich weiterzubilden. Es muss breiter aufgestellt sein (Interview 05, Z. 14).

Damit ist vor allem eine Einbettung von Alphabetisierungskursen in niedrigschwellige Angebote gemeint, wie z. B. Kochkurse oder Umgang mit neuen Medien. Unter dem Deckmantel dieser Themen wird das Ziel verfolgt, mehr Teilnehmende für Alphabetisierungskurse zu gewinnen - zum einen, damit die in der Praxis angebotenen Kurse überhaupt besucht werden und stattfinden können; zum anderen, damit die Förderung nicht ins Leere läuft und in den regelmäßig von den Ländern vorzulegenden Berichten Erfolge sichtbar gemacht werden können. Auch die arbeitsplatzorientierte Ausrichtung von Grundbildung dient dem Ziel, neue Zielgruppen zur Teilnahme an Alphabetisierungskursen zu motivieren.

\section{Diskussion und Fazit}

Die Frage, was eine Grundbildung Erwachsener umfasst, ist nicht unabhängig von den Interessen der beteiligten Akteure sowie den Machtverhältnissen zu verstehen. Im Feld der öffentlichen Bildungsverwaltung spielen neben der Orientierung an allgemeinen Zielen wie Teilhabe und Weiterlernen zudem eine Reihe partikularer, bildungspolitischer Interessen eine Rolle und haben zur Folge, dass Grundbildung auf ganz bestimmte Art und Weise ein- bzw. abgegrenzt wird. Das Interesse der öffentlichen Bildungsverwaltung speist sich dabei insbesondere aus zuständigkeitsbezogenen, finanziellen und erfolgsbezogenen Interessen der Ministerien und Ressorts. Das hat zur Konsequenz, dass Grundbildung zwar angesichts Allgemeinwohl orientierter Ziele über Alphabetisierung hinausgehend gedacht wird, aufgrund förderpolitischer Interessen allerdings eine Verengung auf alltagsbezogene Lese- und Schreibkompetenzen weiterhin dominant bleibt.

Das bedeutet: Es wird zwar von „Grundbildung“ gesprochen und eine Ausweitung von Alphabetisierung angesichts gesellschaftlicher Anforderungen gewünscht, faktisch aber geht es weiterhin im Kern um Alphabetisierung, auch wenn dies so nicht 
offiziell verlautbart wird. Die schwammige Verwendung des Begriffs selbst erfüllt damit die Funktion, eine Vielzahl von Interessen unter einer „Schönwetter-Rhetorik“ zu bündeln, welche einen Scheinkonsens suggeriert und zudem anschlussfähig an internationale Diskurse um ,adult basic education“, „basic skills“ und „,functional skills“ ist. Tatsächlich wird der in der nationalen Strategie geforderte Austausch zwischen unterschiedlichen Akteuren, Ministerien und Ressorts nur bedingt eingelöst, so dass Ein- und Abgrenzungen aufgrund begrenzter Zuständigkeiten und Verantwortlichkeiten kaum überwunden werden können. Bestrebungen, bestehende Handlungsspielräume zu überwinden, um Grundbildung breiter ansetzen zu können, finden sich nur vereinzelt, so beispielsweise in dem Versuch, die Fördermittel des ESF auch auf nicht unmittelbar berufliche, sondern politische oder kulturelle Inhalte auszuweiten.

Die Frage der Abgrenzung von „Grundbildung“ zur „Allgemeinbildung“ sowie zur „Erwachsenen- und Weiterbildung“ ist davon abhängig, wie alle drei Begriffe ausgelegt werden. Allgemeinbildung ist in der Vergangenheit ebenfalls in vielfältiger Weise interpretiert und konzipiert worden und unterliegt einer Reihe differenter Interessen. Bezüglich einer Abgrenzung zur Erwachsenen- und Weiterbildung ist das von Tenorth (2004) dargelegte Stufenmodell hilfreich, das Grundbildung als unterste, vorgelagerte Stufe von Bildungs- und Lernprozessen versteht. Ob dabei allerdings alle Inhalte der Erwachsenenbildung in das Grundbildungskonzept übernommen werden, Grundbildung sich folglich aus den untersten Stufen von politischer, kultureller und beruflicher Erwachsenen- und Weiterbildung zusammensetzt, oder ob nur bestimmte Kompetenzen und Inhalte als Teil einer enger gefassten Grundbildung gedacht werden, ob diese allen oder nur bestimmten Zielgruppen offen steht und ob Übergänge zwischen informellen, non-formalen und formalen Formaten geschaffen werden, ist zwischen den beteiligten Akteuren auszuhandeln.

Dass diese Ein- und Abgrenzungsfragen keineswegs trivial sind, sondern vor allem immer auch mit finanziellen und letztlich existenziellen Interessen zusammenhängen, zeigt auch die Sorge einiger Akteure, der Grundbildungsbegriff könnte den Begriff der breiter gedachten Erwachsenen- und Weiterbildung ersetzen und zu einer fragilen, deutlich reduzierten bildungspolitischen Förderung der Erwachsenenbildung führen - insbesondere dann, wenn Grundbildung möglicherweise zukünftig wieder aus dem Fokus bildungspolitischer Förderung fallen könnte. Trotz dieser Sorge könnte aber als Einwand gelten, dass eine Ausweitung von Grundbildung über Alphabetisierung hinaus unter Einbezug weiterer grundlegender Inhalte und Kompetenzen umso wichtiger wäre, um die in den meisten Bundesländern in Grundzügen gesetzlich gesicherte Weiterbildungsförderung auf möglichst breite Füße zu stellen und zudem Möglichkeiten der häufig vernachlässigten Förderung kultureller und politischer Erwachsenenbildung zu schaffen. Auch stellt eine Annäherung an den Grundbildungsbegriff nicht nur von institutioneller, sondern auch von der Seite der Adressatinnen und Adressaten selbst und deren Bedürfnissen eine sinnvolle Erweiterung der Perspektive dar, um möglichst alle Interessen einzubeziehen.

Zukünftig muss es folglich darum gehen, die diversen Interessen aller beteiligten Akteure transparent $\mathrm{zu}$ machen und in die Diskussion um eine angemessene Grundbildung einzubinden. Gerade angesichts der ressortspezifischen Definition von 
Grundbildung wäre hier eine Koordinierung bzw. zumindest ressortübergreifende Verständigung anzudenken.

Mit Blick auf weiterführende Forschung wäre zu untersuchen, inwieweit sich die für das Feld der öffentlichen Bildungsverwaltung relevanten Interessen und Machtverhältnisse sowie die daraus resultierende Ausdeutung von Grundbildung auch in anderen Feldern bzw. bei anderen Akteuren wiederfinden lassen. Welche Unterschiede und Gemeinsamkeiten gibt es und wie werden diese begründet?

Auch bleibt abzuwarten, welche Konsequenzen sich hieraus für die Gestaltung von Programmen und Angeboten in der Praxis ergeben. Inwieweit kommt es aufgrund der aufgezeigten bildungspolitischen Setzungen zu Verschiebungen der Inhalte, Kompetenzen, Zielgruppen und Angebotsformate einer Grundbildung? Interessante Zugänge sind hier über Ansätze der Programm- bzw. Programmplanungsforschung denkbar, welche auf diese Weise die Frage der Verhältnisbestimmung allgemeiner, beruflicher und politischer Erwachsenen- und Weiterbildung andenken könnten.

Open Access Dieser Artikel wird unter der Creative Commons Namensnennung 4.0 International Lizenz (http://creativecommons.org/licenses/by/4.0/deed.de) veröffentlicht, welche die Nutzung, Vervielfältigung, Bearbeitung, Verbreitung und Wiedergabe in jeglichem Medium und Format erlaubt, sofern Sie den/die ursprünglichen Autor(en) und die Quelle ordnungsgemäß nennen, einen Link zur Creative Commons Lizenz beifügen und angeben, ob Änderungen vorgenommen wurden.

\section{Literatur}

Barton, D., \& Hamilton, M. (2000). Literacy practices. In D. Barton, M. Hamilton \& R. lvanic (Hrsg.), Situated literacies. Reading and writing in context (S. 7-15). London: Routledge.

BMBF \& KMK (2012). Vereinbarung über eine gemeinsame nationale Strategie für Alphabetisierung und Grundbildung Erwachsener in Deutschland 2012-2016. http://www.bmbf.de/pubRD/NEU_ strategiepapier_nationale_alphabetisierung\%281\%29.pdf. Zugegriffen: 27. Januar 2014.

Bourdieu, P. (2005). Was heißt sprechen? Zur Ökonomie des sprachlichen Tausches. Wien: Braumüller.

Bourdieu, P. (2014). Über den Staat. Vorlesungen am Collège de France 1989-1992. Berlin: Suhrkamp.

Bremer, H. (2010). Symbolische Macht und politisches Feld. In B. Lösch \& A. Thimmel (Hrsg.), Kritische politische Bildung. Ein Handbuch (S. 181-192). Schwalbach: Wochenschau.

Döbert, M. (1999). Grundbildung. http://socioweb.leuphana.de/lexikon/lex_geb/begriffe/grundbil.htm. Zugegriffen: 10. Juni 2014.

Egloff, B. (2014). Grundbildung - Zur Einführung in den Themenschwerpunkt. Editorial. Hessische Blätter für Volksbildung, 64(2), 103-106.

Euringer, C. (2016a). Was ist „Grundbildung Erwachsener“? Das Grundbildungsverständnis der öffentlichen Bildungsverwaltung im Kontext von Interessen und Machtverhältnissen. Dissertation. Hamburg: Universität Hamburg.

Euringer, C. (2016b). Grundbildung im Spannungsfeld förderpolitischer Interessen. ALFA-Forum, 89, $36-41$.

Europäische Kommission (2007). Schlüsselkompetenzen für Lebenslanges Lernen. Ein Europäischer Referenzrahmen. http://www.kompetenzrahmen.de/files/europaeischekommission2007de.pdf. Zugegriffen: 12. August 2015.

Faulstich, P., \& Haberzeth, E. (2007). Recht und Politik. Bielefeld: W. Bertelsmann.

Grotlüschen, A. (2010). Erneuerung der Interessetheorie. Wiesbaden: VS.

Grotlüschen, A., \& Riekmann, W. (Hrsg.). (2012). Funktionaler Analphabetismus in Deutschland. Ergebnisse der ersten leo. - Level-One Studie. Münster: Waxmann.

Grotlüschen, A., Heinemann, A., \& Nienkemper, B. (2009). Die unterschätzte Macht legitimer Literalität. REPORT, 32(4), 55-67.

Grotlüschen, A., Bonna, F., Euringer, C., \& Heinemann, A. (2014). Konsequenzen der Konstruktion von Literalität hinsichtlich der Vergleichbarkeit der Alpha-Levels mit den Niveaustufen des Europäischen 
Referenzrahmens Sprachen. In H. Pätzold, H. v. Felden \& S. Schmidt-Lauff (Hrsg.), Programme, Themen und Inhalte in der Erwachsenenbildung (S. 51-65). Baltmannsweiler: Schneider.

Holzkamp, K. (1985). Grundlegung der Psychologie. Frankfurt a. M.: Campus.

Holzkamp, K. (1995). Lernen. Subjektwissenschaftliche Grundlegung. Frankfurt a. M.: Campus.

Hopf, C. (1978). Die Pseudo-Exploration - Überlegungen zur Technik qualitativer Interviews in der Sozialforschung. Zeitschrift für Soziologie, 2, 97-115.

Ioannidou, A. (2010). Steuerung im transnationalen Bildungsraum. Internationales Bildungsmonitoring zum Lebenslangen Lernen. Bielefeld: W. Bertelsmann.

Jann, W. (2008). Regieren als Governance-Problem. In W. Jann \& K. König (Hrsg.), Regieren zu Beginn des 21. Jahrhunderts (S. 1-28). Tübingen: Mohr Siebeck.

Kegelmann, J. (2013). Die Zukunft der Verwaltung. In J. Kegelmann \& K.-U. Martens (Hrsg.), Kommunale Nachhaltigkeit (S. 245-260). Baden-Baden: Nomos.

Koch, S. (2011). Steuerung aus neoinstitutionalistischer Perspektive. In C. Hof, J. Ludwig \& B. Schäffer (Hrsg.), Steuerung, Regulation, Gestaltung. Governance-Prozesse in der Erwachsenenbildung zwischen Struktur und Handlung (S. 81-92). Baltmannsweiler: Schneider.

Linde, A. (2002). Analphabetismus - Alphabetisierung - Grundbildung. Unklarheiten und Gestaltungsräume. In M. Tröster (Hrsg.), Berufsorientierte Grundbildung. Konzepte und Praxishilfen (S. 17-31). Bielefeld: W. Bertelsmann.

Mallows, D., \& Carpentieri, J. D. (2015). Policy coherence. http://www.basicskills.eu/wp-content/uploads/ 2015/06/Transcript.pdf. Zugegriffen: 31. August 2015.

Mey, G., \& Mruck, K. (2010). Interviews. In G. Mey \& K. Mruck (Hrsg.), Handbuch Qualitative Forschung in der Psychologie (S. 423-435). Wiesbaden: Springer VS.

Nienkemper, B. (2015). Lernstandsdiagnostik bei funktionalem Analphabetismus. Akzeptanz und Handlungsstrategien. Bielefeld: W. Bertelsmann.

Rat der Weiterbildung - KAW (2016). Grundbildung als dynamischen Begriff verstehen. Deutsches Institut für Erwachsenenbildung und Rat der Weiterbildung - KAW führten Fachtagung zum Thema Grundbildung durch. http://www.rat-der-weiterbildung.de/wp-content/uploads/2016/04/2016_04_ 20-Pressemitteilung-Fachtagung2.pdf. Zugegriffen: 05. Juli 2016.

Schemmann, M. (2007). Internationale Weiterbildungspolitik und Globalisierung. Orientierungen und Aktivitäten von OECD, EU, UNESCO und Weltbank. Bielefeld: W. Bertelsmann.

Strauss, A.L., \& Corbin, J.M. (1996). Grounded Theory. Grundlagen qualitativer Sozialforschung. Weinheim: Beltz.

Street, B. V. (1992). Sociocultural dimensions of literacy. Literacy in an international context. In UNESCO-Institut für Pädagogik (Hrsg.), The future of literacy and the literacy of the future (S. 41-53). Hamburg: Unesco Institute for Education.

Tenorth, H.-E. (2004). Stichwort: „Grundbildung“ und „Basiskompetenzen“. Herkunft, Bedeutung und Probleme im Kontext allgemeiner Bildung. Zeitschrift für Erziehungswissenschaft, 7(2), 169-182.

Tröster, M. (2000). Grundbildung - Begriffe, Fakten, Orientierungen. In M. Tröster (Hrsg.), Spannungsfeld Grundbildung (S. 12-27). Bielefeld: W. Bertelsmann. 